\title{
Article \\ Positive Solutions of a Fractional Boundary Value Problem with Sequential Derivatives
}

\author{
Alexandru Tudorache ${ }^{1}$ and Rodica Luca ${ }^{2, *(D)}$ \\ 1 Department of Computer Science and Engineering, Gh. Asachi Technical University, 700050 Iasi, Romania; \\ alexandru-gabriel.tudorache@academic.tuiasi.ro \\ 2 Department of Mathematics, Gh. Asachi Technical University, 700506 Iasi, Romania \\ * Correspondence: rluca@math.tuiasi.ro
}

check for

updates

Citation: Tudorache, A.; Luca, R. Positive Solutions of a Fractional Boundary Value Problem with Sequential Derivatives. Symmetry 2021, 13, 1489. https://doi.org/10.3390/ sym13081489

Academic Editor: Calogero Vetro

Received: 17 June 2021

Accepted: 11 August 2021

Published: 13 August 2021

Publisher's Note: MDPI stays neutral with regard to jurisdictional claims in published maps and institutional affiliations.

Copyright: (c) 2021 by the authors. Licensee MDPI, Basel, Switzerland. This article is an open access article distributed under the terms and conditions of the Creative Commons Attribution (CC BY) license (https:// creativecommons.org/licenses/by/ $4.0 /)$.
Abstract: We investigate the existence of positive solutions of a Riemann-Liouville fractional differential equation with sequential derivatives, a positive parameter and a nonnegative singular nonlinearity, supplemented with integral-multipoint boundary conditions which contain fractional derivatives of various orders and Riemann-Stieltjes integrals. Our general boundary conditions cover some symmetry cases for the unknown function. In the proof of our main existence result, we use an application of the Krein-Rutman theorem and two theorems from the fixed point index theory.

Keywords: Riemann-Liouville fractional differential equation; nonlocal boundary conditions; singular functions; positive solutions

MSC: 34A08; 34B10; 34B16; 34B18

\section{Introduction}

In this paper, we consider the ordinary fractional differential equation with sequential derivatives

$$
D_{0+}^{\alpha}\left(q(t) D_{0+}^{\beta} v(t)\right)=\lambda r(t) g(t, v(t)), \quad t \in(0,1),
$$

subject to the integral-multipoint boundary conditions

$$
\left\{\begin{array}{l}
v^{(k)}(0)=0, k=0, \ldots, n-2, D_{0+}^{\beta} v(0)=0, \\
q(1) D_{0+}^{\beta} v(1)=a q(\xi) D_{0+}^{\beta} v(\xi), D_{0+}^{\gamma_{0}} v(1)=\sum_{j=1}^{p} \int_{0}^{1} D_{0+}^{\gamma_{j}} v(t) d \mathcal{H}_{j}(t),
\end{array}\right.
$$

where $\alpha \in(1,2], \beta \in(n-1, n], n \in \mathbb{N}, n \geq 3, p \in \mathbb{N}, \gamma_{i} \in \mathbb{R}, i=0, \ldots, p, 0 \leq \gamma_{1}<\gamma_{2}<$ $\cdots<\gamma_{p} \leq \gamma_{0}<\beta-1, \gamma_{0} \geq 1, \lambda>0, a \geq 0, \xi \in(0,1), q:[0,1] \rightarrow(0, \infty)$ is a continuous function, $g:[0,1] \times(0, \infty) \rightarrow[0, \infty)$ is a continuous function which may have singularity at the second variable in the point 0 , the function $r:(0,1) \rightarrow[0, \infty)$ is continuous and may be singular at $t=0$ and/or $t=1, D_{0+}^{\zeta}$ is the Riemann-Liouville fractional derivative of order $\varsigma$, for $\varsigma=\alpha, \beta, \gamma_{0}, \gamma_{1}, \ldots, \gamma_{p}$, and in (2) we have Riemann-Stieltjes integrals, where $\mathcal{H}_{j}, j=1, \ldots, p$ are bounded variation functions. The general boundary conditions (2) cover some symmetry cases for the unknown function. For example, in the case $p=1$, $\gamma_{0}=\gamma_{1}$ and $\mathcal{H}_{1}$ is a step function given by $\mathcal{H}_{1}(t)=\{0, t=0 ; 1, t \in(0,1]\}$, the last condition from (2) becomes the symmetry condition $D_{0+}^{\gamma_{0}} v(1)=D_{0+}^{\gamma_{0}} v(0)$ for the fractional derivative of order $\gamma_{0}$ of the unknown function $v$. Besides, if $\gamma_{0}=\gamma_{1}=1$, then we find the periodicity condition for the first derivative of function $v$, namely $v^{\prime}(1)=v^{\prime}(0)$.

We present some assumptions on the functions $g$ and $r$, and intervals for the parameter $\lambda$ such that problem (1), (2) has at least one positive solution. By a positive solution of (1), (2) we mean a function $v \in C[0,1]$ satisfying (1) and (2) with $v(t)>0$ for all $t \in(0,1]$. In the proof of our main theorem we use some results from the fixed point index theory. Positive 
solutions for such fractional problems are of great practical importance for describing nonlocal processes with memory, which determines the relevance of the chosen research topic. In what follows we present some recent results connected with our problem (1), (2). In [1], the authors studied the fractional differential equation

$$
D_{0+}^{\gamma} w(t)+\mu h(t) g(t, w(t))=0, \quad t \in(0,1),
$$

subject to nonlocal boundary conditions

$$
w(0)=w^{\prime}(0)=\cdots=w^{(n-2)}(0)=0, \quad D_{0+}^{\zeta_{0}} w(1)=\sum_{i=1}^{m} \int_{0}^{1} D_{0+}^{\zeta_{i}} w(t) d \mathcal{K}_{i}(t),
$$

where $\gamma \in \mathbb{R}, \gamma \in(n-1, n], n \in \mathbb{N}, n \geq 3, m \in \mathbb{N}, \zeta_{j} \in \mathbb{R}$ for all $j=0, \ldots, m$, $0 \leq \zeta_{1}<\zeta_{2}<\cdots<\zeta_{m} \leq \zeta_{0}<\gamma-1, \zeta_{0} \geq 1, \mathcal{K}_{j}, j=1, \ldots, m$ are bounded variation functions, $\mu$ is a positive parameter, the function $g(t, w)$ is nonnegative and it may have singularity at $w=0$ and the function $h(t)$ is nonnegative and it may be singular at the points $t=0$ and/or $t=1$. They gave various assumptions for the functions $h$ and $g$, and established intervals for the parameter $\mu$ such that problem (3), (4) has at least one positive solution. The expression for intervals of $\mu$ are given by using the principal characteristic value of an associated linear operator. The fixed point index theory was used in the proof of the main theorems. By using the Guo-Krasnosel'skii fixed point theorem, a related semipositone problem is also studied in [1]. In [2], the authors studied the system of fractional differential equations with sequential derivatives

$$
\left\{\begin{array}{l}
D_{0+}^{\gamma_{1}}\left(D_{0+}^{\delta_{1}} v(t)\right)+\mu \phi(t, v(t), w(t))=0, \quad t \in(0,1) \\
D_{0+}^{\gamma_{2}}\left(D_{0+}^{\delta_{2}} w(t)\right)+v \psi(t, v(t), w(t))=0, \quad t \in(0,1)
\end{array}\right.
$$

with the coupled boundary conditions

$$
\left\{\begin{array}{l}
v^{(k)}(0)=0, k=0, \ldots, p-2 ; \quad D_{0+}^{\delta_{1}} v(0)=0, \quad D_{0+}^{\alpha_{0}} v(1)=\sum_{j=1}^{n} \int_{0}^{1} D_{0+}^{\alpha_{j}} w(t) d \mathcal{H}_{j}(t), \\
w^{(k)}(0)=0, k=0, \ldots, q-2 ; \quad D_{0+}^{\delta_{2}} w(0)=0, D_{0+}^{\beta_{0}} w(1)=\sum_{j=1}^{m} \int_{0}^{1} D_{0+}^{\beta_{j}} v(t) d \mathcal{K}_{j}(t),
\end{array}\right.
$$

where $\gamma_{1}, \gamma_{2} \in(0,1], \delta_{1} \in(p-1, p], \delta_{2} \in(q-1, q], p, q \in \mathbb{N}, p, q \geq 3, n, m \in \mathbb{N}, \alpha_{j} \in \mathbb{R}$ for all $j=0,1, \ldots, n, 0 \leq \alpha_{1}<\alpha_{2}<\cdots<\alpha_{n} \leq \beta_{0}<\delta_{2}-1, \beta_{0} \geq 1, \beta_{j} \in \mathbb{R}$ for all $j=0,1, \ldots, m, 0 \leq \beta_{1}<\beta_{2}<\cdots<\beta_{m} \leq \alpha_{0}<\delta_{1}-1, \alpha_{0} \geq 1, \mu>0, v>0, \phi$ and $\psi$ are continuous functions which change sign and they may be singular at the points $t=0$ and/or $t=1, \mathcal{H}_{j}, j=1, \ldots, n$ and $\mathcal{K}_{j}, j=1, \ldots, m$ are bounded variation functions. They present various assumptions for the nonsingular/singular functions $\phi$ and $\psi$, and intervals for parameters $\mu$ and $v$ such that problem (5), (6) has at least one or two positive solutions. They applied the nonlinear alternative of Leray-Schauder type and the GuoKrasnosel'skii fixed point theorem in the proofs of the main existence results. We also mention the papers [3-7], and the books [4,8-18] with their references for other results obtained in the last years and for applications of the fractional differential equations and systems in various fields.

The structure of the paper is as follows. In Section 2 we study a linear fractional boundary value problem associated to our problem (1), (2), and we present the associated Green functions with their properties and bounds. Section 3 is concerned with the main existence theorem for (1), (2), and an example illustrating our result is presented in Section 4.

\section{Preliminary Results}

We consider the fractional differential equation

$$
D_{0+}^{\alpha}\left(q(t) D_{0+}^{\beta} v(t)\right)=z(t), \quad t \in(0,1),
$$


with the boundary conditions (2), where $q \in C([0,1],(0, \infty))$ and $z \in C(0,1) \cap L^{1}(0,1)$. We introduce the following numbers

$$
\Delta_{1}=1-a \xi^{\alpha-1}, \Delta_{2}=\frac{\Gamma(\beta)}{\Gamma\left(\beta-\gamma_{0}\right)}-\sum_{i=1}^{p} \frac{\Gamma(\beta)}{\Gamma\left(\beta-\gamma_{i}\right)} \int_{0}^{1} \vartheta^{\beta-\gamma_{i}-1} d \mathcal{H}_{i}(\vartheta) .
$$

Lemma 1. If $\Delta_{1} \neq 0$ and $\Delta_{2} \neq 0$, then problem (2), (7) has the unique solution $v \in C[0,1]$ given by

$$
v(t)=\int_{0}^{1} \mathcal{G}_{2}(t, \vartheta)\left(\frac{1}{q(\vartheta)} \int_{0}^{1} \mathcal{G}_{1}(\vartheta, \zeta) z(\zeta) d \zeta\right) d \vartheta, t \in[0,1]
$$

where

$$
\mathcal{G}_{1}(t, \vartheta)=g_{1}(t, \vartheta)+\frac{a t^{\alpha-1}}{\Delta_{1}} g_{1}(\xi, \vartheta), \quad(t, \vartheta) \in[0,1] \times[0,1],
$$

with

$$
g_{1}(s, \vartheta)=\frac{1}{\Gamma(\alpha)}\left\{\begin{array}{l}
s^{\alpha-1}(1-\vartheta)^{\alpha-1}-(s-\vartheta)^{\alpha-1}, 0 \leq \vartheta \leq s \leq 1 \\
s^{\alpha-1}(1-\vartheta)^{\alpha-1}, 0 \leq s \leq \vartheta \leq 1
\end{array}\right.
$$

and

$$
\mathcal{G}_{2}(t, \vartheta)=g_{2}(t, \vartheta)+\frac{t^{\beta-1}}{\Delta_{2}} \sum_{i=1}^{p}\left(\int_{0}^{1} g_{3 i}(\tau, \vartheta) d \mathcal{H}_{i}(\tau)\right), \quad(t, \vartheta) \in[0,1] \times[0,1]
$$

with

$$
\begin{aligned}
& g_{2}(s, \vartheta)=\frac{1}{\Gamma(\beta)}\left\{\begin{array}{l}
s^{\beta-1}(1-\vartheta)^{\beta-\gamma_{0}-1}-(s-\vartheta)^{\beta-1}, 0 \leq \vartheta \leq s \leq 1, \\
s^{\beta-1}(1-\vartheta)^{\beta-\gamma_{0}-1}, 0 \leq s \leq \vartheta \leq 1,
\end{array}\right. \\
& g_{3 i}(s, \vartheta)=\frac{1}{\Gamma\left(\beta-\gamma_{i}\right)}\left\{\begin{array}{l}
s^{\beta-\gamma_{i}-1}(1-\vartheta)^{\beta-\gamma_{0}-1}-(s-\vartheta)^{\beta-\gamma_{i}-1}, 0 \leq \vartheta \leq s \leq 1, \\
s^{\beta-\gamma_{i}-1}(1-\vartheta)^{\beta-\gamma_{0}-1}, 0 \leq s \leq \vartheta \leq 1 .
\end{array}\right. \\
& i=1, \ldots, p .
\end{aligned}
$$

Proof. We denote by $q(t) D_{0+}^{\beta} v(t)=w(t)$. Then problem (2), (7) is equivalent to the following two boundary value fractional problems

$$
\left\{\begin{array}{l}
D_{0+}^{\alpha} w(t)=z(t), \quad t \in(0,1) \\
w(0)=0, w(1)=a w(\xi)
\end{array}\right.
$$

and

$$
\left\{\begin{array}{l}
D_{0+}^{\beta} v(t)=w(t) / q(t), t \in(0,1) \\
v^{(k)}(0)=0, k=0, \ldots, n-2, D_{0+}^{\gamma_{0}} v(1)=\sum_{j=1}^{p} \int_{0}^{1} D_{0+}^{\gamma_{j}} v(\vartheta) d \mathcal{H}_{j}(\vartheta) .
\end{array}\right.
$$

By Lemma 4.1.5 from [12], problem $(I)$ has the unique solution $w \in C[0,1]$ given by

$$
w(t)=-\int_{0}^{1} \mathcal{G}_{1}(t, \vartheta) z(\vartheta) d \vartheta, \quad t \in[0,1],
$$

where $\mathcal{G}_{1}$ is defined in (10). By Lemma 2.4.2 from [9], problem (II) has the unique solution $v \in C[0,1]$ given by

$$
v(t)=-\int_{0}^{1} \mathcal{G}_{2}(t, \vartheta) w(\vartheta) / q(\vartheta) d \vartheta, \quad t \in[0,1],
$$

where $\mathcal{G}_{2}$ is defined in (12). Now by using (14) and (15) we obtain the solution $v$ for problem (2), (7) which is given by relation (9). 
By applying some properties of functions $g_{1}, g_{2}, g_{3 i}, i=1, \ldots, p$ presented in (11) and $(13)$ (see $[9,12])$, we deduce the following result.

Lemma 2. If $\mathcal{H}_{j}, j=1, \ldots, p$ are nondecreasing functions, $\Delta_{1}>0, \Delta_{2}>0$, then the Green functions $\mathcal{G}_{1}$ and $\mathcal{G}_{2}$ given by (10) and (12) have the following properties:

(a) $\mathcal{G}_{1}, \mathcal{G}_{2}:[0,1] \times[0,1] \rightarrow[0, \infty)$ are continuous functions;

(b) $\mathcal{G}_{1}(t, \vartheta) \leq \mathcal{J}_{1}(\vartheta), \forall(t, \vartheta) \in[0,1] \times[0,1]$, where

$$
\mathcal{J}_{1}(\vartheta)=h_{1}(\vartheta)+\frac{a}{\Delta_{1}} g_{1}(\xi, \vartheta), \forall \vartheta \in[0,1],
$$

and $h_{1}(\vartheta)=\frac{1}{\Gamma(\alpha)}(1-\vartheta)^{\alpha-1}, \vartheta \in[0,1]$

(c) $\mathcal{G}_{2}(t, \vartheta) \leq \mathcal{J}_{2}(\vartheta), \forall(t, \vartheta) \in[0,1] \times[0,1]$, where

$$
\mathcal{J}_{2}(\vartheta)=h_{2}(\vartheta)+\frac{1}{\Delta_{2}} \sum_{i=1}^{p} \int_{0}^{1} g_{3 i}(\tau, \vartheta) d \mathcal{H}_{i}(\tau), \forall \vartheta \in[0,1],
$$

and $h_{2}(\vartheta)=\frac{1}{\Gamma(\beta)}(1-\vartheta)^{\beta-\gamma_{0}-1}\left(1-(1-\vartheta)^{\gamma_{0}}\right), \vartheta \in[0,1]$.

(d) $\mathcal{G}_{2}(t, \vartheta) \geq t^{\beta-1} \mathcal{J}_{2}(\vartheta), \forall(t, \vartheta) \in[0,1] \times[0,1]$.

By using Lemma 2, we easily obtain the following lemma (see also [12]).

Lemma 3. If $\Delta_{1}>0, \Delta_{2}>0, \mathcal{H}_{j}, j=1, \ldots, p$ are nondecreasing functions, and $z \in C(0,1) \cap$ $L^{1}(0,1)$ with $z(s) \geq 0$ for all $s \in(0,1)$, then the solution $v$ of problem (2), (7) given by (9) satisfies the properties $v(s) \geq 0$ for all $s \in[0,1]$ and $v(s) \geq t^{\beta-1} v(\eta)$ for all $s, \eta \in[0,1]$.

\section{Existence of Positive Solutions}

In this section we present some conditions on the functions $g$ and $r$, and intervals for $\lambda$ such that there exists at least one positive solution of (1), (2). We introduce the Banach space $\mathcal{X}=C[0,1]$ with supremum norm $\|v\|=\sup _{s \in[0,1]}|v(s)|$, and we introduce the cones

$$
\mathcal{C}=\{v \in \mathcal{X}, v(s) \geq 0, \forall s \in[0,1]\}, \mathcal{S}=\left\{v \in \mathcal{X}, v(s) \geq s^{\beta-1}\|v\|, \forall s \in[0,1]\right\} \subset \mathcal{C} .
$$

We define the operator $\mathcal{E}: \mathcal{C} \rightarrow \mathcal{C}$ and the linear operator $\mathcal{F}: \mathcal{X} \rightarrow \mathcal{X}$ by

$$
\begin{aligned}
& \mathcal{E} v(t)=\lambda \int_{0}^{1} \mathcal{G}_{2}(t, \vartheta) \frac{1}{q(\vartheta)}\left(\int_{0}^{1} \mathcal{G}_{1}(\vartheta, \tau) r(\tau) g(\tau, v(\tau)) d \tau\right) d \vartheta, t \in[0,1], v \in \mathcal{C}, \\
& \mathcal{F} v(t)=\int_{0}^{1} \mathcal{G}_{2}(t, \vartheta) \frac{1}{q(\vartheta)}\left(\int_{0}^{1} \mathcal{G}_{1}(\vartheta, \tau) r(\tau) v(\tau) d \tau\right) d \vartheta, t \in[0,1], v \in \mathcal{X} .
\end{aligned}
$$

We observe that $v$ is a solution of problem (1), (2) if and only if $v$ is a fixed point of operator $\mathcal{E}$. For $\theta>0$ we introduce the sets $\mathcal{S}_{\theta}=B_{\theta} \cap \mathcal{S}$ and $\overline{\mathcal{S}}_{\theta}=\bar{B}_{\theta} \cap \mathcal{S},\left(B_{\theta}=\{v \in \mathcal{X},\|v\|<\theta\}\right.$, $\bar{B}_{\theta}=\{v \in \mathcal{X},\|v\| \leq \theta\}, \partial B_{\theta}=\{v \in \mathcal{X},\|v\|=\theta\}$.

We give now the assumptions that we will use in this section.

(H1) $\alpha \in(1,2], \beta \in(n-1, n], n \in \mathbb{N}, n \geq 3, p \in \mathbb{N}, \gamma_{i} \in \mathbb{R}, i=0, \ldots, p, 0 \leq \gamma_{1}<\gamma_{2}<$ $\cdots<\gamma_{p} \leq \gamma_{0}<\beta-1, \gamma_{0} \geq 1, \lambda>0, a \geq 0, \xi \in(0,1), q:[0,1] \rightarrow(0, \infty)$ is a continuous function, $\mathcal{H}_{i}:[0,1] \rightarrow \mathbb{R}, i=1, \ldots, p$ are nondecreasing functions, $\lambda>0$, and $\Delta_{1}>0, \Delta_{2}>0\left(\Delta_{1}, \Delta_{2}\right.$ are given by (8)).

(H2) The function $r \in C((0,1),[0, \infty))$ and $0<\int_{0}^{1} r(\vartheta) d \vartheta<\infty$. 
(H3) The function $g \in C([0,1] \times(0, \infty),[0, \infty))$ and for any $0<\theta_{1}<\theta_{2}$ we have

$$
\lim _{m \rightarrow \infty} \sup _{v \in \overline{\mathcal{S}}_{\theta_{2}} \backslash \mathcal{S}_{\theta_{1}}} \int_{I_{m}} r(\vartheta) g(\vartheta, v(\vartheta)) d \vartheta=0
$$

where $I_{m}=\left[0, \frac{1}{m}\right] \cup\left[\frac{m-1}{m}, 1\right]$.

Lemma 4. We suppose that assumptions (H1)-(H3) hold. Then for any $0<\theta_{1}<\theta_{2}$, the operator $\mathcal{E}: \overline{\mathcal{S}}_{\theta_{2}} \backslash \mathcal{S}_{\theta_{1}} \rightarrow \mathcal{S}$ is a completely continuous operator.

Proof. By using $(H 3)$, we find that there exists a number $m_{1} \geq 3$ with the property

$$
\sup _{v \in \overline{\mathcal{S}}_{\theta_{2}} \backslash \mathcal{S}_{\theta_{1}}} \int_{I_{m_{1}}} r(\vartheta) g(\vartheta, v(\vartheta)) d \vartheta<1 .
$$

For $v \in \overline{\mathcal{S}}_{\theta_{2}} \backslash \mathcal{S}_{\theta_{1}}$, we find that there exists $\omega_{0} \in\left[\theta_{1}, \theta_{2}\right]$ such that $\|v\|=\omega_{0}$, and then

$$
t^{\beta-1} \theta_{1} \leq t^{\beta-1} \omega_{0} \leq v(t) \leq \omega_{0} \leq \theta_{2}, \forall t \in[0,1] .
$$

Let $M_{1}=\max \left\{g(t, y), t \in\left[\frac{1}{m_{1}}, \frac{m_{1}-1}{m_{1}}\right], y \in\left[\frac{1}{m_{1}^{\beta-1}} \theta_{1}, \theta_{2}\right]\right\}$. By Lemma 2, (H2), and (16), we deduce

$$
\begin{aligned}
& \sup _{v \in \overline{\mathcal{S}}_{\theta_{2}} \backslash \mathcal{S}_{\theta_{1}}} \mathcal{E} v(t)=\sup _{v \in \overline{\mathcal{S}}_{\theta_{2}} \backslash \mathcal{S}_{\theta_{1}}} \lambda \int_{0}^{1} \mathcal{G}_{2}(t, \vartheta) \frac{1}{q(\vartheta)}\left(\int_{0}^{1} \mathcal{G}_{1}(\vartheta, \tau) r(\tau) g(\tau, v(\tau)) d \tau\right) d \vartheta \\
& \leq \sup _{v \in \overline{\mathcal{S}}_{\theta_{2}} \backslash \mathcal{S}_{\theta_{1}}} \lambda \int_{0}^{1} \mathcal{J}_{2}(\vartheta) \frac{1}{q(\vartheta)}\left(\int_{0}^{1} \mathcal{J}_{1}(\tau) r(\tau) g(\tau, v(\tau)) d \tau\right) d \vartheta \\
& \leq \sup _{v \in \overline{\mathcal{S}}_{\theta_{2}} \backslash \mathcal{S}_{\theta_{1}}} \lambda \int_{0}^{1} \mathcal{J}_{2}(\vartheta) \frac{1}{q(\vartheta)}\left(\int_{I_{m_{1}}} \mathcal{J}_{1}(\tau) r(\tau) g(\tau, v(\tau)) d \tau\right) d \vartheta \\
& +\sup _{v \in \overline{\mathcal{S}}_{\theta_{2}} \backslash \mathcal{S}_{\theta_{1}}} \lambda \int_{0}^{1} \mathcal{J}_{2}(\vartheta) \frac{1}{q(\vartheta)}\left(\int_{\frac{1}{m_{1}}}^{\frac{m_{1}-1}{m_{1}}} \mathcal{J}_{1}(\tau) r(\tau) g(\tau, v(\tau)) d \tau\right) d \vartheta \\
& \leq \lambda J_{10} \int_{0}^{1} \mathcal{J}_{2}(\vartheta) \frac{1}{q(\vartheta)} d \vartheta+\lambda M_{1}\left(\int_{0}^{1} \mathcal{J}_{2}(\vartheta) \frac{1}{q(\vartheta)} d \vartheta\right)\left(\int_{\frac{1}{m_{1}}}^{\frac{m_{1}-1}{m_{1}}} \mathcal{J}_{1}(\tau) r(\tau) d \tau\right) \\
& \leq \lambda J_{10}\left(\int_{0}^{1} \mathcal{J}_{2}(\vartheta) \frac{1}{q(\vartheta)} d \vartheta\right)\left(1+M_{1} \int_{0}^{1} r(\tau) d \tau\right)<\infty,
\end{aligned}
$$

where $J_{10}=\max _{s \in[0,1]} \mathcal{J}_{1}(s)>0$. This gives us that the operator $\mathcal{E}$ is well defined.

Next we show that $\mathcal{E}: \overline{\mathcal{S}}_{\theta_{2}} \backslash \mathcal{S}_{\theta_{1}} \rightarrow \mathcal{S}$. Indeed, for any $v \in \overline{\mathcal{S}}_{\theta_{2}} \backslash \mathcal{S}_{\theta_{1}}$ and $t \in[0,1]$, we have

$$
\begin{aligned}
& \mathcal{E} v(t)=\lambda \int_{0}^{1} \mathcal{G}_{2}(t, \vartheta) \frac{1}{q(\vartheta)}\left(\int_{0}^{1} \mathcal{G}_{1}(\vartheta, \tau) r(\tau) g(\tau, v(\tau)) d \tau\right) d \vartheta \\
& \leq \lambda \int_{0}^{1} \mathcal{J}_{2}(\vartheta) \frac{1}{q(\vartheta)}\left(\int_{0}^{1} \mathcal{G}_{1}(\vartheta, \tau) r(\tau) g(\tau, v(\tau)) d \tau\right) d \vartheta
\end{aligned}
$$

and then

$$
\|\mathcal{E} v\| \leq \lambda \int_{0}^{1} \mathcal{J}_{2}(\vartheta) \frac{1}{q(\vartheta)}\left(\int_{0}^{1} \mathcal{G}_{1}(\vartheta, \tau) r(\tau) g(\tau, v(\tau)) d \tau\right) d \vartheta .
$$

By Lemma 2, we also obtain

$$
\begin{aligned}
& \mathcal{E} v(t) \geq \lambda t^{\beta-1} \int_{0}^{1} \mathcal{J}_{2}(\vartheta) \frac{1}{q(\vartheta)}\left(\int_{0}^{1} \mathcal{G}_{1}(\vartheta, \tau) r(\tau) g(\tau, v(\tau)) d \tau\right) d \vartheta \\
& \geq t^{\beta-1}\|\mathcal{E} v\|, \quad \forall t \in[0,1],
\end{aligned}
$$


hence $\mathcal{E} v \in \mathcal{S}$. Then $\mathcal{E}\left(\overline{\mathcal{S}}_{\theta_{2}} \backslash \mathcal{S}_{\theta_{1}}\right) \subset \mathcal{S}$.

We prove now that $\mathcal{E}: \overline{\mathcal{S}}_{\theta_{2}} \backslash \mathcal{S}_{\theta_{1}} \rightarrow \mathcal{S}$ is a completely continuous operator. We assume that $D \subset \overline{\mathcal{S}}_{\theta_{2}} \backslash \mathcal{S}_{\theta_{1}}$ is an (arbitrary) bounded set. By using the first part of the proof, we deduce that $\mathcal{E}(D)$ is uniformly bounded. We show next that $\mathcal{E}(D)$ is equicontinuous. For $\epsilon>0$ there exists a natural number $m_{2} \geq 3$ satisfying the condition

$$
\sup _{v \in \overline{\mathcal{S}}_{\theta_{2}} \backslash \mathcal{S}_{\theta_{1}}} \int_{I_{m_{2}}} r(\vartheta) g(\vartheta, \vartheta(\vartheta)) d \vartheta<\frac{\epsilon}{4 \lambda J_{10}}\left(\int_{0}^{1} \frac{\mathcal{J}_{2}(\vartheta)}{q(\vartheta)} d \vartheta\right)^{-1} .
$$

Because $\mathcal{G}_{2}(s, \vartheta)$ is uniformly continuous on $[0,1] \times[0,1]$, then for the above $\epsilon>0$ we deduce that there exists $\rho>0$ such that for any $t_{1}, t_{2} \in[0,1]$ with $\left|t_{1}-t_{2}\right|<\rho$ and $\vartheta \in[0,1]$ we have

$$
\left|\mathcal{G}_{2}\left(t_{1}, \vartheta\right)-\mathcal{G}_{2}\left(t_{2}, \vartheta\right)\right|<\frac{\epsilon}{2 \lambda r_{0} J_{10} M_{2}}\left(\int_{0}^{1} \frac{1}{q(\vartheta)} d \vartheta\right)^{-1} .
$$

Here $M_{2}=\max \left\{1, \max \left\{g(t, y), t \in\left[\frac{1}{m_{2}}, \frac{m_{2}-1}{m_{2}}\right], y \in\left[\frac{1}{m_{2}^{\beta-1}} \theta_{1}, \theta_{2}\right]\right\}\right\}$ and $r_{0}=\max \{1$, $\left.\max \left\{r(\vartheta), \vartheta \in\left[\frac{1}{m_{2}}, \frac{m_{2}-1}{m_{2}}\right]\right\}\right\}$.

Therefore for any $v \in D, t_{1}, t_{2} \in[0,1]$ with $\left|t_{1}-t_{2}\right|<\rho$, we find

$$
\begin{aligned}
& \left|(\mathcal{E} v)\left(t_{1}\right)-(\mathcal{E} v)\left(t_{2}\right)\right| \\
& =\lambda\left|\int_{0}^{1}\left(\mathcal{G}_{2}\left(t_{1}, \vartheta\right)-\mathcal{G}_{2}\left(t_{2}, \vartheta\right)\right) \frac{1}{q(\vartheta)}\left(\int_{0}^{1} \mathcal{G}_{1}(\vartheta, \tau) r(\tau) g(\tau, v(\tau)) d \tau\right) d \vartheta\right| \\
& \leq 2 \lambda \sup _{v \in D} \int_{0}^{1} \mathcal{J}_{2}(\vartheta) \frac{1}{q(\vartheta)}\left(\int_{I_{m_{2}}} \mathcal{J}_{1}(\tau) r(\tau) g(\tau, v(\tau)) d \tau\right) d \vartheta \\
& +\lambda \sup _{v \in D} \int_{0}^{1}\left|\mathcal{G}_{2}\left(t_{1}, \vartheta\right)-\mathcal{G}_{2}\left(t_{2}, \vartheta\right)\right| \frac{1}{q(\vartheta)}\left(\int_{\frac{1}{m_{2}}}^{\frac{m_{2}-1}{m_{2}}} \mathcal{J}_{1}(\tau) r(\tau) g(\tau, v(\tau)) d \tau\right) d \vartheta \\
& \leq \frac{\epsilon}{2}+\frac{\epsilon}{2}=\epsilon .
\end{aligned}
$$

So we obtain that $\mathcal{E}(D)$ is equicontinuous. By the Arzela-Ascoli theorem, we deduce that $\mathcal{E}: \overline{\mathcal{S}}_{\theta_{2}} \backslash \mathcal{S}_{\theta_{1}} \rightarrow \mathcal{S}$ is compact.

We prove next that $\mathcal{E}: \overline{\mathcal{S}}_{\theta_{2}} \backslash \mathcal{S}_{\theta_{1}} \rightarrow \mathcal{S}$ is continuous. We assume that $v_{n}, v_{0} \in \overline{\mathcal{S}}_{\theta_{2}} \backslash \mathcal{S}_{\theta_{1}}$ for all $n \geq 1$, and $\left\|v_{n}-v_{0}\right\| \rightarrow 0$ as $n \rightarrow \infty$. Then $\theta_{1} \leq\left\|v_{n}\right\| \leq \theta_{2}$ for all $n \geq 0$. By $(H 3)$, for $\epsilon>0$ there exists a natural number $m_{3} \geq 3$ satisfying the condition

$$
\sup _{v \in \overline{\mathcal{S}}_{\theta_{2}} \backslash \mathcal{S}_{\theta_{1}}} \int_{I_{m_{3}}} r(\vartheta) g(\vartheta, v(\vartheta)) d \vartheta<\frac{\epsilon}{4 \lambda J_{10}}\left(\int_{0}^{1} \mathcal{J}_{2}(\vartheta) \frac{1}{q(\vartheta)} d \vartheta\right)^{-1} .
$$

Because $g$ is uniformly continuous in $\left[\frac{1}{m_{3}}, \frac{m_{3}-1}{m_{3}}\right] \times\left[\frac{1}{m_{3}^{\beta-1}} \theta_{1}, \theta_{2}\right]$, we find

$$
\lim _{n \rightarrow \infty}\left|g\left(\vartheta, v_{n}(\vartheta)\right)-g\left(\vartheta, v_{0}(\vartheta)\right)\right|=0, \text { uniformly for } \vartheta \in\left[\frac{1}{m_{3}}, \frac{m_{3}-1}{m_{3}}\right] .
$$

Therefore by using the Lebesgue dominated convergence theorem, we deduce

$$
\int_{\frac{1}{m_{3}}}^{\frac{m_{3}-1}{m_{3}}} r(\vartheta)\left|g\left(\vartheta, v_{n}(\vartheta)\right)-g\left(\vartheta, v_{0}(\vartheta)\right)\right| d \vartheta \rightarrow 0, \text { as } n \rightarrow \infty .
$$

So, for the above $\epsilon>0$ there exists another natural number $m_{4}$ such that for all $n>m_{4}$ we obtain

$$
\int_{\frac{1}{m_{3}}}^{\frac{m_{3}-1}{m_{3}}} r(\vartheta)\left|g\left(\vartheta, v_{n}(\vartheta)\right)-g\left(\vartheta, v_{0}(\vartheta)\right)\right| d \vartheta<\frac{\epsilon}{2 \lambda J_{10}}\left(\int_{0}^{1} \mathcal{J}_{2}(\vartheta) \frac{1}{q(\vartheta)} d \vartheta\right)^{-1} .
$$


By (17) and (18) we conclude that

$$
\begin{aligned}
& \left\|\mathcal{E} v_{n}-\mathcal{E} v_{0}\right\| \\
& \leq \sup _{v \in \overline{\mathcal{S}}_{\theta_{2}} \backslash \mathcal{S}_{\theta_{1}}} \lambda \int_{0}^{1} \mathcal{J}_{2}(\vartheta) \frac{1}{q(\vartheta)}\left(\int_{I_{m_{3}}} \mathcal{J}_{1}(\tau) r(\tau)\left|g\left(\tau, v_{n}(\vartheta)\right)-g\left(\tau, v_{0}(\tau)\right)\right| d \tau\right) d \vartheta \\
& \quad+\sup _{v \in \overline{\mathcal{S}}_{\theta_{2}} \backslash \mathcal{S}_{\theta_{1}}} \lambda \int_{0}^{1} \mathcal{J}_{2}(\vartheta) \frac{1}{q(\vartheta)}\left(\int_{\frac{1}{m_{3}}}^{\frac{m_{3}-1}{m_{3}}} \mathcal{J}_{1}(\tau) r(\tau)\left|g\left(\tau, v_{n}(\tau)\right)-g\left(\tau, v_{0}(\tau)\right)\right| d \tau\right) d \vartheta \\
& \leq \frac{\epsilon}{2}+\frac{\epsilon}{2}=\epsilon, \quad \forall n>m_{4} .
\end{aligned}
$$

So we find that $\mathcal{E}: \overline{\mathcal{S}}_{\theta_{2}} \backslash \mathcal{S}_{\theta_{1}} \rightarrow \mathcal{S}$ is a continuous operator. Therefore $\mathcal{E}$ is a completely continuous operator.

Under assumptions $(H 1)-(H 3)$, by using the extension theorem, the operator $\mathcal{E}$ has a completely continuous extension (we also denoted it by $\mathcal{E}$ ) from $\mathcal{S}$ to $\mathcal{S}$.

By using the Krein-Rutman theorem in the space $C[0,1]$ and similar methods as those used in the proof of Lemma 3.2 from [1], we obtain the following lemma.

Lemma 5. We suppose that assumptions $(H 1)$ and $(H 2)$ hold. Then the spectral radius $r(\mathcal{F}) \neq 0$ and $\mathcal{F}$ has an eigenfunction $\zeta_{1} \in \mathcal{C} \backslash\{0\}$ which corresponds to the principal eigenvalue $r(\mathcal{F})$, that is $\mathcal{F} \zeta_{1}=r(\mathcal{F}) \zeta_{1}$. So $r(\mathcal{F})>0$.

By using a similar approach as that used in the proof of Lemma 4 for operator $\mathcal{E}$, we deduce that $\mathcal{F}(\mathcal{S}) \subset \mathcal{S}$.

Theorem 1. We suppose that assumptions (H1)-(H3) hold. If

$$
0 \leq \mathfrak{g}_{\infty}^{s}:=\limsup _{y \rightarrow \infty} \max _{t \in[0,1]} \frac{g(t, y)}{y}<\mathfrak{g}_{0}^{i}:=\liminf _{y \rightarrow 0+} \min _{t \in[0,1]} \frac{g(t, y)}{y} \leq \infty,
$$

then for any $\lambda \in\left(\frac{1}{\mathfrak{g}_{0}^{i} r(\mathcal{F})}, \frac{1}{\mathfrak{g}_{\infty}^{\text {s }} r(\mathcal{F})}\right)$ there exists at least one positive solution $v(t), t \in[0,1]$ of problem (1), (2), (we use here the conventions $\frac{1}{0+}=\infty$ and $\frac{1}{\infty}=0+$ ).

Proof. Let $\lambda \in\left(\frac{1}{\mathfrak{g}_{0}^{i} r(\mathcal{F})}, \frac{1}{\mathfrak{g}_{\infty}^{s} r(\mathcal{F})}\right)$. For $\mathfrak{g}_{0}^{i}$, there exists $\omega_{1}>0$ such that $g(\tau, y) \geq \frac{y}{\lambda r(\mathcal{F})}$ for all $\tau \in[0,1]$ and $y \in\left[0, \omega_{1}\right]$. Hence for any $v \in \partial \mathcal{S}_{\omega_{1}}$ we obtain

$$
\begin{aligned}
& \mathcal{E} v(t)=\lambda \int_{0}^{1} \mathcal{G}_{2}(t, \vartheta) \frac{1}{q(\vartheta)}\left(\int_{0}^{1} \mathcal{G}_{1}(\vartheta, \tau) r(\tau) g(\tau, v(\tau)) d \tau\right) d \vartheta \\
& \geq \frac{1}{r(\mathcal{F})} \int_{0}^{1} \mathcal{G}_{2}(t, \vartheta) \frac{1}{q(\vartheta)}\left(\int_{0}^{1} \mathcal{G}_{1}(\vartheta, \tau) r(\tau) v(\tau) d \tau\right) d \vartheta=\frac{1}{r(\mathcal{F})} \mathcal{F} v(t), \quad \forall t \in[0,1] .
\end{aligned}
$$

We suppose that $\mathcal{E}$ has no fixed point on $\partial \mathcal{S}_{\omega_{1}}$ (if not, the proof is finished). We will prove that

$$
v-\mathcal{E} v \neq v \zeta_{1}, \forall v \in \partial \mathcal{S}_{\omega_{1}}, \quad v \geq 0,
$$

where $\zeta_{1}$ is given in Lemma 5. We assume that there exist $v_{1} \in \partial \mathcal{S}_{\omega_{1}}$ and $v_{1} \geq 0$ such that $v_{1}-\mathcal{E} v_{1}=v_{1} \zeta_{1}$. Then $v_{1}>0$ and $v_{1}=\mathcal{E} v_{1}+v_{1} \zeta_{1} \geq v_{1} \zeta_{1}$. We denote by $v_{0}=$ $\sup \left\{v, v_{1} \geq v \zeta_{1}\right\}$. Then $v_{0} \geq v_{1}, v_{1} \geq v_{0} \zeta_{1}$ and

$$
\mathcal{E} v_{1} \geq \frac{1}{r(\mathcal{F})} \mathcal{F} v_{1} \geq \frac{1}{r(\mathcal{F})} v_{0} \mathcal{F} \zeta_{1}=v_{0} \zeta_{1}
$$


So $v_{1}=\mathcal{E} v_{1}+v_{1} \zeta_{1} \geq v_{0} \zeta_{1}+v_{1} \zeta_{1}=\left(v_{0}+v_{1}\right) \zeta_{1}$, which represents a contradiction with the definition of $v_{0}$. Then relation (19) holds, and by [19] we conclude that

$$
i\left(\mathcal{E}, \mathcal{S}_{\omega_{1}}, \mathcal{S}\right)=0
$$

For $\mathfrak{g}_{\infty}^{s}$, we deduce that there exist $\eta \in(0,1)$ and $\omega_{2}>\omega_{1}$ such that $g(\tau, y) \leq \eta \frac{1}{\lambda r(\mathcal{F})} y$ for all $\tau \in[0,1]$ and $y \in\left[\omega_{2}, \infty\right)$. We introduce the linear operator $\mathcal{F}_{1}: \mathcal{X} \rightarrow \mathcal{X}$ by

$$
\mathcal{F}_{1} v(t)=\eta \frac{1}{r(\mathcal{F})} \mathcal{F} v(t)=\frac{\eta}{r(\mathcal{F})} \int_{0}^{1} \mathcal{G}_{2}(t, \vartheta) \frac{1}{q(\vartheta)}\left(\int_{0}^{1} \mathcal{G}_{1}(\vartheta, \tau) r(\tau) v(\tau) d \tau\right) d \vartheta,
$$

for all $t \in[0,1]$ and $v \in \mathcal{X}$. The above operator $\mathcal{F}_{1}$ is bounded, and $\mathcal{F}_{1}(\mathcal{S}) \subset \mathcal{S}$. Because $\eta \in(0,1)$ we find $r\left(\mathcal{F}_{1}\right)=\eta<1$. We consider the set $\mathcal{U}=\left\{v \in \mathcal{S} \backslash B_{\omega_{1}}, v v=\right.$ $\mathcal{E} v$ with $v \geq 1\}$. For $v \in \mathcal{S}$, we denote by $K(v)=\left\{t \in[0,1], v(t) \geq \omega_{2}\right\}$. Then for $v \in \mathcal{S}$, we have $v(t) \geq \omega_{2}$ for all $t \in K(v)$, and so

$$
g(t, v(t)) \leq \eta \frac{1}{\lambda r(\mathcal{F})} v(t), \quad \forall t \in K(v) .
$$

By relation (21) and the definition of linear operator $\mathcal{F}$, we find for any $v \in \mathcal{U}, v \geq 1$ and $t \in[0,1]$ that

$$
\begin{aligned}
v(t) \leq v v(t)=(\mathcal{E} v)(t)=\lambda \int_{0}^{1} \mathcal{G}_{2}(t, \vartheta) \frac{1}{q(\vartheta)}\left(\int_{0}^{1} \mathcal{G}_{1}(\vartheta, \tau) r(\tau) g(\tau, v(\tau)) d \tau\right) d \vartheta \\
=\lambda \int_{0}^{1} \mathcal{G}_{2}(t, \vartheta) \frac{1}{q(\vartheta)}\left(\int_{K(v)} \mathcal{G}_{1}(\vartheta, \tau) r(\tau) g(\tau, v(\tau)) d \tau\right) d \vartheta \\
\quad+\lambda \int_{0}^{1} \mathcal{G}_{2}(t, \vartheta) \frac{1}{q(\vartheta)}\left(\int_{[0,1] \backslash K(v)} \mathcal{G}_{1}(\vartheta, \tau) r(\tau) g(\tau, v(\tau)) d \tau\right) d \vartheta \\
\leq \frac{\eta}{r(\mathcal{F})} \int_{0}^{1} \mathcal{G}_{2}(t, \vartheta) \frac{1}{q(\vartheta)}\left(\int_{K(v)} \mathcal{G}_{1}(\vartheta, \tau) r(\tau) v(\tau) d \tau\right) d \vartheta \\
\quad+\lambda \int_{0}^{1} \mathcal{J}_{2}(\vartheta) \frac{1}{q(\vartheta)}\left(\int_{0}^{1} \mathcal{J}_{1}(\tau) r(\tau) g(\tau, \widetilde{v}(\tau)) d \tau\right) d \vartheta \\
\leq \frac{\eta}{r(\mathcal{F})} \int_{0}^{1} \mathcal{G}_{2}(t, \vartheta) \frac{1}{q(\vartheta)}\left(\int_{0}^{1} \mathcal{G}_{1}(\vartheta, \tau) r(\tau) v(\tau) d \tau\right) d \vartheta \\
\quad+\lambda J_{10}\left(\int_{0}^{1} \mathcal{J}_{2}(\vartheta) \frac{1}{q(\vartheta)} d \vartheta\right) M_{3}=\mathcal{F}_{1} v(t)+\lambda J_{10}\left(\int_{0}^{1} \mathcal{J}_{2}(\vartheta) \frac{1}{q(\vartheta)} d \vartheta\right) M_{3},
\end{aligned}
$$

where $\widetilde{v}(\tau)=\min \left\{v(\tau), \omega_{2}\right\}$ for any $\tau \in[0,1]$; this function satisfies the inequalities

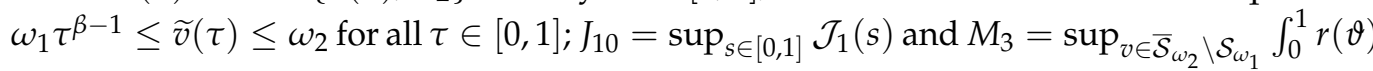
$\times g(\vartheta, v(\vartheta)) d \vartheta$, (as in the proof of Lemma 4 we find that $M_{3}<\infty$ ). By the Gelfand formula we know that $\left(I-\mathcal{F}_{1}\right)^{-1}$ exists and $\left(I-\mathcal{F}_{1}\right)^{-1}=\sum_{i=1}^{\infty} \mathcal{F}_{1}^{i}$, which gives us $\left(I-\mathcal{F}_{1}\right)^{-1}(\mathcal{S}) \subset \mathcal{S}$. This together with (22) imply

$$
v(t) \leq\left(I-\mathcal{F}_{1}\right)^{-1}\left(\lambda J_{10} M_{3} \int_{0}^{1} \mathcal{J}_{2}(\vartheta) \frac{1}{q(\vartheta)} d \vartheta\right), \forall t \in[0,1],
$$

and so

$$
v(t) \leq \lambda J_{10} M_{3}\left(\int_{0}^{1} \mathcal{J}_{2}(\vartheta) \frac{1}{q(\vartheta)} d \vartheta\right)\left\|\left(I-\mathcal{F}_{1}\right)^{-1}\right\|, \quad \forall t \in[0,1],
$$

which implies that the set $\mathcal{U}$ is bounded. We choose now $\omega_{3}>\max \left\{\omega_{2}, \sup \{\|v\|, v \in \mathcal{U}\}\right\}$. We find $v v \neq \mathcal{E} v$ for all $v \in \partial \mathcal{S}_{\omega_{3}}$ and $v \geq 1$. By [19], we deduce that

$$
i\left(\mathcal{E}, \mathcal{S}_{\omega_{3}}, \mathcal{S}\right)=1
$$

By (20), (23) and the properties of the fixed point index, we conclude that

$$
i\left(\mathcal{E}, \mathcal{S}_{\omega_{3}} \backslash \overline{\mathcal{S}}_{\omega_{1}}, \mathcal{S}\right)=i\left(\mathcal{E}, \mathcal{S}_{\omega_{3}}, \mathcal{S}\right)-i\left(\mathcal{E}, \mathcal{S}_{\omega_{1}}, \mathcal{S}\right)=1
$$


Then operator $\mathcal{E}$ has at least one fixed point on $\mathcal{S}_{\omega_{3}} \backslash \overline{\mathcal{S}}_{\omega_{1}}$ which represents a positive solution of problem (1), (2).

\section{An Example}

Let $\alpha=\frac{4}{3}, \beta=\frac{7}{2}(n=4), p=2, a=\frac{11}{10}, \xi=\frac{1}{3}, \gamma_{0}=\frac{9}{4}, \gamma_{1}=\frac{1}{6}, \gamma_{2}=\frac{5}{3}, q(\tau)=\frac{1}{\tau+1}$ for all $\tau \in[0,1], H_{1}(\tau)=\frac{\tau}{4}$ for all $\tau \in[0,1], H_{2}(\tau)=\left\{\frac{1}{3}, \tau \in\left[0, \frac{1}{2}\right) ; \frac{14}{9}, \tau \in\left[\frac{1}{2}, 1\right]\right\}$, $r(\tau)=\frac{1}{\sqrt[4]{\tau(1-\tau)^{3}}}$ for all $\tau \in(0,1), g(\tau, x)=\sqrt[3]{x}+\tau^{2}+\frac{1}{\sqrt[7]{x^{2}}}$ for all $\tau \in[0,1]$ and $x>0$.

We consider the fractional differential equation

$$
D_{0+}^{4 / 3}\left(\frac{1}{t+1} D_{0+}^{7 / 2} v(t)\right)=\frac{\lambda}{\sqrt[4]{t(1-t)^{3}}}\left(\sqrt[3]{v(t)}+t^{2}+\frac{1}{\sqrt[7]{v^{2}(t)}}\right), t \in(0,1)
$$

with the boundary conditions

$$
\left\{\begin{array}{l}
v(0)=v^{\prime}(0)=v^{\prime \prime}(0)=0, D_{0+}^{7 / 2} v(0)=0, D_{0+}^{7 / 2} v(1)=\frac{33}{20} D_{0+}^{7 / 2} v\left(\frac{1}{3}\right), \\
D_{0+}^{9 / 4} v(1)=\frac{1}{4} \int_{0}^{1} D_{0+}^{1 / 6} v(t) d t+\frac{11}{9} D_{0+}^{5 / 3} v\left(\frac{1}{2}\right) .
\end{array}\right.
$$

We have $\Delta_{1} \approx 0.23730259>0$ and $\Delta_{2} \approx 1.15334282>0$, and so assumption (H1) is satisfied. In addition we find $\int_{0}^{1} r(\vartheta) d \vartheta \approx 4.44288294 \in(0, \infty)$, and then assumption $(H 2)$ is also satisfied.

$$
\begin{aligned}
& \text { For } 0<\theta_{1}<\theta_{2}, v \in \overline{\mathcal{S}}_{\theta_{2}} \backslash \mathcal{S}_{\theta_{1}} \text { and } I_{m}=\left[0, \frac{1}{m}\right] \cup\left[\frac{m-1}{m}, 1\right] \text {, we obtain } \\
& \Lambda_{m}=\int_{I_{m}} r(\vartheta) g(\vartheta, v(\vartheta)) d \vartheta=\int_{I_{m}} \frac{1}{\sqrt[4]{\vartheta(1-\vartheta)^{3}}}\left(\sqrt[3]{\vartheta(\vartheta)}+\vartheta^{2}+\frac{1}{\sqrt[7]{v^{2}(\vartheta)}}\right) d \vartheta \\
& \leq \int_{I_{m}} \frac{1}{\sqrt[4]{\vartheta(1-\vartheta)^{3}}}\left(\sqrt[3]{\theta_{2}}+1+\frac{1}{\sqrt[7]{\left(\vartheta^{5 / 2} \theta_{1}\right)^{2}}}\right) d \vartheta \\
& =\left(\sqrt[3]{\theta_{2}}+1\right) \int_{I_{m}} \frac{d \vartheta}{\sqrt[4]{\vartheta(1-\vartheta)^{3}}}+\frac{1}{\sqrt[7]{\theta_{1}^{2}}} \int_{I_{m}} \frac{1}{\vartheta^{27 / 28}(1-\vartheta)^{3 / 4}} d \vartheta
\end{aligned}
$$

and then $\lim _{m \rightarrow \infty} \sup _{v \in \overline{\mathcal{S}}_{\theta_{2}} \backslash \mathcal{S}_{\theta_{1}}} \Lambda_{m}=0$, because $g_{1}(\vartheta)=\frac{1}{\vartheta^{1 / 4}(1-\vartheta)^{3 / 4}} \in L^{1}(0,1)$ and $g_{2}(\vartheta)=\frac{1}{\vartheta^{27 / 28}(1-\vartheta)^{3 / 4}} \in L^{1}(0,1)$. So assumption (H3) is satisfied. In addition we find $\mathfrak{g}_{\infty}^{s}=0$ and $\mathfrak{g}_{0}^{i}=\infty$. Then by applying Theorem 1 , we conclude that for any $\lambda \in(0, \infty)$ there exists at least one positive solution $v(t), t \in[0,1]$ of problem (24), (25), satisfying the condition $v(t) \geq t^{5 / 2}\|v\|$ for all $t \in[0,1]$.

\section{Conclusions}

In this paper we study the fractional differential Equation (1) with sequential derivatives and a positive parameter, subject to general nonlocal boundary conditions (2) containing Riemann-Stieltjes integrals and fractional derivatives of various orders. The function $g$ from the equation is a nonnegative continuous function and it may be singular at the second variable in the point 0 , and the function $r$ is also nonnegative continuous one and it may be singular at the points $t=0$ and $t=1$. The general boundary conditions (2) cover some symmetry cases (as periodicity conditions for derivatives) for the unknown function. By using an application of the Krein-Rutman theorem in the space $C[0,1]$, and some theorems from the fixed point index theory, we prove that problem (1), (2) has at least one positive solution $v(t), t \in[0,1]$. To illustrate our main existence theorem, we finally present an example. 
Author Contributions: Conceptualization, R.L.; Formal analysis, A.T. and R.L.; Methodology, A.T. and R.L. Both authors have read and agreed to the published version of the manuscript.

Funding: This research received no external funding.

Institutional Review Board Statement: Not applicable.

Informed Consent Statement: Not applicable.

Data Availability Statement: Not applicable.

Conflicts of Interest: The authors declare no conflict of interest.

\section{References}

1. Tudorache, A.; Luca, R. On a singular Riemann-Liouville fractional boundary value problem with parameters. Nonlinear Anal. Model. Control 2021, 26, 151-168. [CrossRef]

2. Henderson, J.; Luca, R.; Tudorache, A. Positive solutions for a system of coupled semipositone fractional boundary value problems with sequential fractional derivatives. Mathematics 2021, 9, 753. [CrossRef]

3. Ahmad, B.; Alsaedi, A.; Ntouyas, S.K.; Shammakh, W. On more general boundary value problems involving sequential fractional derivatives. Adv. Differ. Equ. 2019, 290, 1-25. [CrossRef]

4. Fazli, H.; Nieto, J.J.; Bahrami, F. On the existence and uniqueness results for nonlinear sequential fractional differential equations. Appl. Comput. Math. 2018, 17, 36-47.

5. $\mathrm{Su}, \mathrm{X} . ;$ Zhang, S.; Zhang, L. Periodic boundary value problem involving sequential fractional derivatives in Banach space. AIMS Math. 2020, 5, 7510-7530. [CrossRef]

6. Wei, Z.; Li, Q.; Che, J. Initial value problems for fractional differential equations involving Riemann-Liouville sequential fractional derivative. J. Math. Anal. Appl. 2010, 367, 260-272. [CrossRef]

7. Ye, H.; Huang, R. Initial value problem for nonlinear fractional differential equations with sequential fractional derivative. Adv. Differ. Equ. 2015, 291, 1-13. [CrossRef]

8. Ahmad, B.; Alsaedi, A.; Ntouyas, S.K.; Tariboon, J. Hadamard-Type Fractional Differential Equations, Inclusions and Inequalities; Springer: Cham, Switzerland, 2017.

9. Ahmad, B.; Henderson, J.; Luca, R. Boundary Value Problems for Fractional Differential Equations and Systems, Trends in Abstract and Applied Analysis 9; World Scientific: Hackensack, NJ, USA, 2021.

10. Baleanu, D.; Diethelm, K.; Scalas, E.; Trujillo, J.J. Fractional Calculus Models and Numerical Methods; Series on Complexity, Nonlinearity and Chaos; World Scientific: Boston, MA, USA, 2012.

11. Das, S. Functional Fractional Calculus for System Identification and Controls; Springer: New York, NY, USA, 2008.

12. Henderson, J.; Luca, R. Boundary Value Problems for Systems of Differential, Difference and Fractional Equations. Positive Solutions; Elsevier: Amsterdam, The Netherlands, 2016.

13. Kilbas, A.A.; Srivastava, H.M.; Trujillo, J.J. Theory and Applications of Fractional Differential Equations, North-Holland Mathematics Studies, 204; Elsevier Science B.V.: Amsterdam, The Netherlands, 2006.

14. Klafter, J.; Lim, S.C.; Metzler, R. (Eds.) Fractional Dynamics in Physics; World Scientific: Singapore, 2011.

15. Podlubny, I. Fractional Differential Equations; Academic Press: San Diego, CA, USA, 1999.

16. Sabatier, J.; Agrawal, O.P.; Machado, J.A.T. (Eds.) Advances in Fractional Calculus: Theoretical Developments and Applications in Physics and Engineering; Springer: Dordrecht, The Netherlands, 2007.

17. Samko, S.G.; Kilbas, A.A.; Marichev, O.I. Fractional Integrals and Derivatives. Theory and Applications; Gordon and Breach: Yverdon, Switzerland, 1993.

18. Zhou, Y. Basic Theory of Fractional Differential Equations; World Scientific: Singapore, 2014.

19. Amann, H. Fixed point equations and nonlinear eigenvalue problems in ordered Banach spaces. SIAM Rev. 1976, 18, 620-709. [CrossRef] 